\title{
The Effectiveness of Mindfulness Therapy in Controlling under Treatment Addicts' Drug Cravings
}

\author{
Bahram Iranshahri ${ }^{1}$, Hossein Jenaabadi ${ }^{*}$ \\ ${ }^{1}$ MA. Educational Psychology, Department of Psychology, Zahedan Branch, Islamic Azad University, Zahedan, Iran \\ ${ }^{2}$ Department of Education, University of Sistan and Baluchestan, Zahedan, Iran \\ Email: ${ }^{*}$ jenaabadi@ped.usb.ac.ir
}

Received 23 November 2014; accepted 2 April 2015; published 22 July 2015

Copyright (C 2015 by authors and Scientific Research Publishing Inc.

This work is licensed under the Creative Commons Attribution International License (CC BY). http://creativecommons.org/licenses/by/4.0/

c) (i) Open Access

\begin{abstract}
The present study aimed to examine the effectiveness of mindfulness therapy in controlling under treatment addicts' drug cravings. This was a quasi-experimental study with pretest and posttest. The sample of this study included 30 under treatment male addicts whom were selected through applying random sampling method and were placed in two groups, experimental and control. After holding a preliminary session aiming at aiding the experimental group to become familiar with the procedure, 8 sessions of the mindfulness-based therapy were administered. Assessment tools were Craving Beliefs Questionnaire (CBQ) and the Depression Anxiety Stress Scale (DASS-21). After calculating mean and standard deviation, the obtained data were analyzed using covariance. The results indicated that the mindfulness therapy was significantly effective in controlling drug cravings, decreasing stress, anxiety, and depression of under treatment addicts $(p<0.05)$.
\end{abstract}

\section{Keywords}

Mindfulness-Based Stress Management Treatment, Drug Cravings, Depression, Anxiety, Stress, Addiction

\section{Introduction}

Drug dependence is accompanied with craving. Drug craving is considered as a key concept in relapse, drug abuse, and recurrence. Craving is regarded synonymous with ask for, impulses, desires, need or compulsion to drug abuse. Craving is a self-conscious experience of desire to use drugs [1].

\footnotetext{
${ }^{*}$ Corresponding author.
}

How to cite this paper: Iranshahri, B. and Jenaabadi, H. (2015) The Effectiveness of Mindfulness Therapy in Controlling under Treatment Addicts' Drug Cravings. Open Journal of Medical Psychology, 4, 88-98. 
The Tenth International Classification of Diseases considered craving as the first criterion for diagnosis of drug dependence. By creating attentional bias and selective information processing, through associating with signs, processing related information in a relatively more appealing manner, increasing distractions, reducing the ability of concentrating on triggers, strengthening the awareness of internal states, and decelerating perception and the passage of time, craving can affect recurrence of drug abuse [2].

Most drug addicts, during their addiction, struggle with drug rehabilitation; however, after a while, they relapse and continue their drug abuse. Dealing with addicts' mental problems including restoration of self-esteem, self-confidence, responsibility, and social and familial issues is directly correlated with drug rehabilitation [3]. The researchers concluded that three-quarters of those who had completed their treatment relapsed within a year after completion of the treatment [4].

Among new methods of drug treatment, especially psychological treatments, integrating mindfulness mediation techniques (mindfulness) with traditional cognitive behavioral therapies can be mentioned, the combination of which is referred to as the third wave of behavioral therapy [5].

Considering the effectiveness of mindfulness-based therapies in decreasing physical and psychological disorders, it seems that this therapy is effective in alleviating some symptoms of the relapse of opioid abuse. Examples of these new approaches are Mindfulness-Based Relapse Prevention (MBRP) [6] and MindfulnessBased Cognitive Therapy (MBCT) to prevent recurrence of depression and drug abuse [7].

Kabat-Zinn's approach for the treatment of drug abuse includes bodily check or technological knowledge of the body and entails a series of deliberate alternations which emphasize the whole body at the beginning and afterwards considers every part of the body without judgment [5]. This method of treatment aims at aiding patients' to apply methods of responding to life stress through which they can emancipate from psychological reactions that can worsen their stress and interfere with the problem-solving methods.

Mindfulness is also used in drug abuse treatments and has positive and significant treatment effects and results. When prevention trainings are combined with mindfulness, they can bring relative success and affect injecting drug users' judgment and aversion. Drug craving is the most powerful predicator of relapse among other predicators (even comorbidity with disorders such as anxiety and depression) and mindfulness can significantly decrease negative effects of craving. The more the severity of mindfulness related to spirituality is, the less the use of alcohol, tobacco and drugs will be [8].

The main objective of the current study was to examine the effectiveness of stress management training based on mindfulness in controlling under treatment addicts' craving.

Hamedi, Shahidi, and Khademi examined the effectiveness of mindfulness therapy and counseling in decreasing harms in preventing the recurrence of drug abuse. In this quasi-experimental study, 90 male addicts who had detoxified during the recent week were selected among all patients in the detoxification and addiction treatment centers in Tehran and were placed in three groups of mindfulness, behavioral counseling aiming at reducing the harm of AIDS and drug abuse, and control. Two weeks after completion of the intervention and two month after posttest, the follow-up test was conducted [9].

Aghayousefi, Oraki, Zare', and Imani conducted a study to investigate the effectiveness of mindfulness therapy in reducing anxiety, depression and stress of drug addicts. Using a quasi-experimental design with pretest and posttest, among all patients referred to Medical Council Clinic in Shahryar and Andishe Rahaee Addition Withdrawal Clinic, 60 addicts were selected through applying convenience sampling method and placed in two groups of control and experimental. Subjects' level of anxiety, depression, and stress was initially evaluated. Afterwards, 8 two-hour sessions were administered on the experimental group and the control group received no such intervention. After conducting the treatment program, subjects' level of anxiety, depression, and stress was assessed again. The results of multivariate covariance analysis indicated that there was a significant difference between the results of pretest and posttest of the experimental group. In this regard, it can be noted that mindfulness training was effective in reducing anxiety, depression, and stress of drug addicts [10].

The results of Sterg, Kazemi, and Raeesi, entitled "The Effectiveness of Cognitive Therapy on Craving Beliefs and Ideas Related to Patients Dependent to Industrial Drugs", showed that cognitive therapy had a significant impact on modifying craving attitudes and beliefs related to drug in both dependent to crack and heroin groups [11].

Zarei, Asadi, and Zarei investigated the effectiveness of stress management training programs on quality of life of addicts under treatment with methadone. The sample included 30 addicts under methadone treatment referred to the MMT center in Pakdasht whom were selected through using random sampling method and were 
randomly divided into two groups of control (15 addicts) and experimental (15 individuals). The experimental group received 10 2-hour sessions of stress management training and the control group received no such treatment. The results demonstrated that the mean score of the experimental group on quality of life was significantly higher than that of the control group. It can be concluded that stress management training program was effective in increasing the level of quality of life of addicts under methadone treatment [12].

Kiani, Ghasemi, and Pourabbas carried out a study entitled "Comparing the Effectiveness of Group Psychotherapy Based on Acceptance, Commitment, and Mindfulness in the Level of Addiction Cravings and Cognitive Emotion Regulation in Methamphetamine Users". The sample included 34 methamphetamine users. The results showed that group psychotherapy had a significant impact on addiction craving in both pretest and posttest. The researchers concluded that the treatment which is based on acceptance, commitment, and mindfulness, since they have common treatment components, reduces the mental health issues caused due to amphetamine abuse [13].

Lak, Moazedian, Hosseini Madani, Sedaghat, and Ameri examined the effectiveness of stress management training using a cognitive-behavioral method in decreasing anxiety of addicts suffering from generalized anxiety disorders. Applying convenience sampling method, 24 addicts who obtained high scores on the Generalized Anxiety Inventory and a questionnaire on anxiety, depression, and stress and were diagnosed with generalized anxiety disorder were selected. The results of covariance analysis indicated the effectiveness of stress management training using behavioral-cognitive therapy in decreasing anxiety and generalized anxiety of the experimental group compared to the control group [14].

Narimani and Rajabi examined the effect of EFG biofeedback on reducing anxiety, depression, stress, and craving beliefs of people with morphine abuse disorders. After 20 sessions of neurofeedback treatment, the obtained results demonstrated its significant impacts on anxiety, depression and craving beliefs; however, considering the variable of stress, no significant difference was found. This study indicated that addicts can improve their anxiety, depression and craving beliefs under the EEG biofeedback [15].

Kiamarsi and Abolghasemi investigated the relationship of self-efficacy, sensation seeking, and strategies of coping with stress with university students' potential for addiction. This was a correlational study. The results of the Pearson correlation test indicated that self-efficacy, sensation-seeking, problem-focused coping strategies, and emotion-focused coping strategies were significantly correlated with university students' addiction potential. The results of multiple regression analysis showed that self-efficacy, sensation-seeking, coping strategies can predict university students' addiction potential [16].

Firozabadi, Ghanbari hashemabadi, and Tabatabaee examined the effectiveness of detached mindfulness techniques and distraction methods in the level of addiction craving and the possibility of relapse in male addicts. The results demonstrated that both methods reduced the subjects' level of addiction craving and affected the possibility of relapse in these individuals [17].

Dabaghi, Asgharnejad Farid, Atef Vahid, and Bolahri conducted a study to investigate the effectiveness of mindfulness-based relapse prevention. Using an experimental design, 90 male patients, whom were diagnosed with opioid dependence based on DSM-IV criteria, were selected after detoxification and were placed into two experimental groups of cognitive behavioral therapy and mindfulness-based relapse prevention and a control group. The results indicated that both methods were more effective in reducing the possibility of their relapse, increasing treatment compliance, length of staying in the treatment, decreasing the physical symptoms and anxiety, and improving their social function compared to Naltrexone. The researchers concluded that using mindfulness-based cognitive relapse prevention can significantly increase the effectiveness of medical therapy and cognitive behavior therapy for treating opioid dependence and also improve patients' mental health [18].

Garland's study conducted on the effectiveness of the mindfulness-based therapy indicated that mindfulness training can increase the cognitive control on addiction craving and decrease stress associated with alcohol abuse; therefore, it can play a key role in alcohol abuse treatment [19].

Garland, Boettiger, Gaylord, West, Chanon, and Howard demonstrated that mindfulness is correlated with controlling attention and emotion regulation. In this regard, increasing the control over the visual clues of drug and alcohol abuse can be effective in treating these disorders [20].

A study conducted by Bowen et al. on the effectiveness of mindfulness relapse prevention indicated that people who had received mindfulness intervention and did detoxification had less relapse within 4 months [21].

In another study, Witkiewitz, Marlatt, and Walker, through examining the effectiveness of mindfulness therapy on prisoners' alcohol rehabilitation treatment, indicated that mindfulness therapy can play a key role in 
treating alcoholic prisoners and controlling their generalized anxiety, compromising an important part of the problems of the people who are trying to withdraw their addiction [7].

The main objective of the current study was to examine the effectiveness of stress management training based on mindfulness in controlling under treatment addicts' craving.

\section{Methods}

The present study, considering its objectives, was an applicable quasi-experimental study with pretest-posttest randomized group design.

The population of this study consisted of all addicts whom were detoxified in addiction centers (Addiction Withdrawal Camp) in Iranshahr. Among three addiction withdrawal clinics, the sample was selected through applying convenience sampling method. A total number of 30 individuals were selected and divided into two groups of control and experimental.

Besides considering all moral conditions, the inclusion criteria of the current study include:

1) Having informed consent to participate in the research;

2) Being detoxified during the last week;

3) Being male, being at least 18 years old and at most 45 years old;

4) Not participating in any other medical sessions;

5) Not suffering from mental retardation or severe disabilities;

6) Not using drugs.

The control group was selected through informing patients about the fact that it takes two months to start the treatment and the experimental group was selected based on spending a one-month time period to initiate the treatment. To eliminate confounding variables, in camps and clinics, participants were selected among those who did not participate in their ordinary treatment sessions. Both groups completed Craving Beliefs Questionnaire (CBQ) and the Depression Anxiety Stress Scale (DASS-21). Afterwards, mindfulness-based stress management training was administered for 8 2-hour sessions. At the end of the sessions, the questionnaires were completed again by the participants.

\subsection{Research Instruments}

Craving Beliefs Questionnaire (CBQ): This is a self-report questionnaire which was developed by Beck et al. (1993), evaluating beliefs related to addiction craving, and has 20 items that are scored based on a 7-point Likert type scale (from totally disagree to completely agree). Reliability and validity of this questionnaire was reported desirable [22]. Considering the conducted studies carried out to examine the relationship of the level of craving and attentional bias towards related stimuli with drug abuse, to investigate the reliability of this questionnaire, the Pearson correlation coefficient was used which was -0.28 . For assessing its reliability, Cronbach's alpha and split half method were used, the results of which were 0.84 and 0.81 , respectively [23].

The short-form of the Depression Anxiety Stress Scale (DASS-21): This is a self-report scale developed by S. H. Lovibond and P. F. Lovinond. It has 21 items and three scales for each indicator under study and is designed to measure the negative emotional states of depression, anxiety and stress. The depression scale assesses depression, feelings of restlessness and blame, despair, devaluing life, self-dissatisfaction, lack of interest/involvement, and immobility. The anxiety scale measures automatic arousal, situational anxiety, and subjective experience of anxiety. The stress scale is sensitive to the levels of chronic arousal which cause difficulty in achieving peace and evaluates nerve impulses and being easily confused, irritability and impatience. Respondents use a 4-point Likert type scale ranges from 0 to $3(0=$ never, $1=$ low, $2=$ moderate, and $3=$ high $)$ to assess experiencing these states in the previous week. The scores on depression, anxiety, and stress are calculated by the sum of the scores on related items [24]. In a study conducted on a normal sample, the results indicated that considering depression, anxiety, and stress, this test had a high internal consistency $(0.91,0.84$, and 0.90 , respectively) [25]. In another study carried out on a clinical population, the internal consistency coefficients (Cronbach's alpha) of these three subscales were calculated which were $0.96,0.89$, and 0.93 , respectively [26]. All of these coefficients were higher than the minimum acceptable amount recommended by Nunnally and Bernstein, i.e. 0.70 [27]. Test-retest coefficients (Pearson) for depression, anxiety, and stress were 0.84, 0.89, and 0.90, respectively. These correlation coefficients were significant $(p<0.001)$. Considering intra-class correlation $(0.74)$, it can be noted that this scale's validity is desirable. Moreover, given the fact that correlation coefficients are significant, it can be mentioned that DASS's reliability is acceptable. 


\subsection{Mindfulness-Based Stress Management}

Mindfulness-based stress management program is administered in 8 2-hour sessions. These 8 intervention sessions were performed based on mindfulness-based stress reduction program.

\subsection{Sessions of Training Mindfulness Technique}

\section{Procedure of the Preliminary Session}

Objectives: Introducing the instructor to the members of the group, introducing MBSR program, becoming familiar with each other and determining the dates of the sessions.

This session initiated with introducing the instructor of the training program. Afterwards, a brief summary of the program and the method of holding it were presented. Then, the instructor emphasized the participants' commitment to attend the sessions, their active participation, and confidentiality. Moreover, doing homework was discussed as an essential and important element of the program. In this session, participants became familiar with each other in a way that each participant introduced himself using a real name or a nickname. In addition to introducing himself, each participant should mention the names of other participants who introduced themselves prior to him. Finally, a preliminary pamphlet entitled "Stress Reduction based on Mindfulness" was given to each participant to study.

Procedure of the First Session

Objectives: Realizing some terms including distraction, automatic guidance, mindfulness, eating meditation training to train the presence of mind, and bodily check meditation to determine every part of the body.

In this session, initially, some terms including distraction, automatic guidance, mindfulness and awareness were explained. Participants could ask their questions related to taught contents. Participants found out that to control the everyday distraction loop and to get away from automatic guidance, they should practice mindfulness. To this end, they were trained (using an eating meditation training) with eating raisins. Afterwards, the issue was discussed and the essential feedbacks were given. The reasons for the use of the body as the first part were presented and it was explained that how higher levels of awareness of body parts can aid participants to control their emotions. The practice of checking body parts was done. Finally, a commitment form, focusing on reducing stress based on mindfulness, listening to the body check CD for 6 days a week, eating a meal with the presence of mind, doing 9 dots exercise and doing their homework presented in their mindfulness pamphlet, was handed over to the participants.

\section{Procedure of the Second Session}

Objectives: Increasing the awareness and following the instructions and training how to deal with challenges with which an individual encounters.

Initially, to achieve mindfulness, bodily check exercise was conducted. This exercise was reviewed and what was essential was taught. Participants realized how anxiety and depression create systematic thoughts and feelings which reinforce each other, creating a vicious cycle which is difficult to deal with. Participants were helped to see this cycle more clearly. At the end, their homework was reviewed and checked and pamphlets of "Breathing with Mindfulness" were distributed among the participants. Pleasant event calendars were given to the participants to write their pleasant events in the time interval between the second and third sessions in order to become more aware of them.

\section{Procedure of the Third Session}

Objectives: Training and gaining the ability to delay judgment, identify stress symptoms, and gain knowledge of mind and body.

In this session, automatic physical, emotional, and behavioral reactions were described using pictures and short and long-term emotions, cognition, behaviors and various physiological aspects of two different responses were discussed. Participants completed a self-report stress checklist to develop their knowledge of various methods through which stress can affect their emotions, feelings and behaviors. Instructions which shift the attention to breathing using mindfulness were given to the participants. A sitting meditation exercise was conducted for 25 minutes and bodily check exercise accompanied with the first chain of physical yoga postures was performed. A member's experiences were presented. Completing the calendar of unpleasant events, doing sitting meditation, listening to the $\mathrm{CD}$ of bodily check with the first yoga postures were proposed as their homework.

Procedure of the Fourth Session

Objectives: Acquiring the ability to practice sitting meditation and listening to the environmental sounds. 
In this session, initially, a preliminary exercise with the experience of seeing and hearing was conducted for 5 minutes to prepare the participants to take part in the sitting meditation and listening to the CD. Additionally, bodily check meditation using the first chain of yoga postures was practiced. Afterwards, their homework was reviewed and essential feedbacks were given. In the next step, their homework and their report were discussed. Finally, their homework including listening to the bodily check CD accompanied with yoga, doing sitting meditation exercises (20 minutes a day) and reading the pamphlet of "How to Deal with Emotional Pains: You Are Not Your Pain But You Can Do Much to Improve It" were distributed among the participants.

\section{Procedure of the Fifth Session}

Objectives: Expanding their knowledge to other areas including physical sensations, sounds, and thoughts or feelings or undetermined purposes.

In this session, a brief summary of sitting meditation exercises with breathing, sitting meditation exercises with breathing and body as a whole, and sitting meditation accompanied with sounds, thoughts and feelings, and undetermined knowledge was proposed. Afterwards, previous exercises were reviewed and the essential feedbacks were given. 3-minute breathing space exercise which focuses on integration and expansion of knowledge were conducted. Homework related to the fourth session was discussed and one of the members read his report. Meditation was performed during walking (walking with mindfulness). Finally, homework, including listening to the sitting meditation CD and reading pamphlets of "Doctors, Patients, and People: Moving towards Integration of Perspectives, Mind, and Body", was presented.

\section{Procedure of the Sixth Session}

Objectives: Reviewing the exercises and experiences of the participants

In this session, sitting meditation which is accompanied with yoga was conducted for 45 minutes and previous exercises were reviewed and participants' homework was proposed which included doing sitting meditation accompanied with yoga and reading the pamphlet of "Stress Caused by Food" which were given to the participants to aid them to become familiar with eating and mindfulness.

\section{Procedure of the Seventh Session}

Objectives: Combing sitting meditation, bodily check, and yoga exercises

In this session, after participants talked about their experiences of doing homework, they conducted the sitting meditation exercise for 15 minutes and the yoga exercise for 30 minutes. As homework, they were asked to do bodily check, sitting meditation and yoga exercises with or without audio tape. Participants were asked to focus on integration of mindfulness in their everyday life.

\section{Procedure of the Eighth Session}

Objectives: Using CDs and becoming ready for doing these exercises alone.

The obtained data was analyzed in both descriptive and inferential levels. In the descriptive level, frequency, percentage, mean, and standard deviation were used and in the inferential level, to examine the research questions, Pearson correlation coefficient and simultaneous multiple regression analysis were applied using $\mathrm{SPSS}_{21}$.

\section{Results}

In this section, demographic information of the participants is summarized. This information includes participants' age and their level of education.

\subsection{Participants' Age Status}

Table 1 compared participants' mean age in the mindfulness treatment group and the control group. Considering the results presented in the above table, the amount of the calculated $\mathrm{T}$ is 0.232 and its probability level is 0.74 which is higher than 0.05 . Therefore, it can be argued that there is no significant difference between the mindfulness treatment group and the control group considering their age.

Table 1. Descriptive data related to participants' age.

\begin{tabular}{cccc}
\hline Groups & N & Mean & SD \\
\hline Mindfulness group therapy & 15 & 29.60 & 3.019 \\
Control group & 15 & 29.33 & 3.26 \\
T value & 0.232 & Probability level & 0.704 \\
\hline
\end{tabular}




\subsection{Participants' Level of Education}

The results presented in Table 2 indicate that the amount of the calculated $\mathrm{T}$ is 0.145 and its probability level is 0.886 which is higher than 0.05 . Therefore, it can be argued that there is no significant difference between the mindfulness group therapy and the control group considering their level of education.

\subsection{Results of Data Analysis Conducted to Test the Hypotheses}

\section{Descriptive and Analytical Results Related to the Craving Beliefs Questionnaire (CBQ)}

In this section, descriptive and analytical results obtained from the Craving Beliefs Questionnaire are presented.

With regard to Table 3, these two groups' mean scores on craving beliefs are almost equal. Considering the results obtained from posttest, there is a difference between these two groups' mean scores indicating that the effectiveness of mindfulness therapy.

\section{Results of Covariance Analysis of Craving Beliefs}

The results demonstrated in Table 5 indicate that conducted interventions significantly change the participants' scores on the Craving Beliefs Questionnaire $(p<0.005)$. In this regard, it can be argued that interventions decrease the participants' scores on craving beliefs. Eta-squared shows that 35 percent of the variance of craving beliefs can be determined by the independent variable that is the mindfulness group therapy.

\section{Analytical Results}

First Hypothesis: There is a significant difference between the effectiveness of mindfulness-based stress management treatment and the control group considering craving beliefs.

With respect to the calculated F-value in Table 4, i.e. $\mathrm{F}(27,1)=13.545, P<0.05)$, the obtained level of significance is 0.000 which is lower than 0.05 . Hence, the null hypothesis is rejected. Accordingly, it can be concluded that there is a significant difference between these two groups' mean scores at the 95 confidence level and the first hypothesis is confirmed.

Descriptive and Analytical Results Related to the Short-Form of the Depression Anxiety Stress Scale (DASS-21)

In this section, descriptive and analytical results obtained from the short-form of the Depression Anxiety Stress Scale (DASS-21) are presented.

Table 2. Descriptive data related to participants' level of education.

\begin{tabular}{cccc}
\hline Groups & $\mathrm{N}$ & Mean & SD \\
\hline Mindfulness group therapy & 15 & 10.13 & 1.12 \\
Control group & 15 & 10.07 & 1.38 \\
T value & 0.145 & Probability level & 0.886 \\
\hline
\end{tabular}

Table 3. Descriptive data related to the Craving Beliefs Questionnaire (CBQ).

\begin{tabular}{|c|c|c|c|}
\hline Tests & Groups & Mean & $\mathrm{SD}$ \\
\hline \multirow{2}{*}{ Pretest } & Mindfulness treatment group & 87.20 & 5.609 \\
\hline & Control group & 89.93 & 6.135 \\
\hline \multirow{2}{*}{ Posttest } & Mindfulness treatment group & 69.80 & 10.276 \\
\hline & Control group & 83.27 & 7.025 \\
\hline
\end{tabular}

Table 4. A summary of the results of covariance analysis of the results obtained from the craving beliefs questionnaire.

\begin{tabular}{cccccc}
\hline Resources of change & Sum of squares (type III) & df & Mean square & F & The probability level \\
\hline Craving beliefs & 969.224 & 1 & 969.224 & 13.545 & 0.001 \\
Main effect & 407.529 & 1 & 407.529 & 6.245 & 0.019 \\
Residual error & 1761.804 & 27 & & & 0.18 \\
\hline
\end{tabular}




\section{Descriptive Results}

Considering the results indicated in Table 5, in the pretest, the participants' mean scores on anxiety, depression, and stress are almost equal. However, in the posttest, the experimental and the control groups' mean scores are different showing the effectiveness of mindfulness therapy.

\section{Results of Covariance Analysis of DASS-21}

The results demonstrated in Table 6 indicate that conducted interventions significantly change the participants' scores on DASS-21 $(p<0.005)$. In this regard, it can be argued that interventions decrease the scores of participants placed in the mindfulness group therapy on anxiety, depression, and stress. Eta-squared shows that 35 percent of the variance of anxiety, 73 percent of the variance of depression, and 77 percent of the variance of stress can be determined by the independent variable that is the mindfulness group therapy.

\section{Analytical Results}

Second Hypothesis: There is a significant difference between the effectiveness of mindfulness-based stress management treatment and the control group in reducing stress.

With respect to the calculated $\mathrm{F}$-value in Table 6, i.e. $\mathrm{F}(27,1)=94.446, P<0.05)$, the obtained level of significance is 0.000 which is lower than 0.05 . Hence, the null hypothesis is rejected. Accordingly, it can be concluded that there is a significant difference between these two groups at the 95 confidence level and the second hypothesis is confirmed.

Third Hypothesis: There is a significant difference between the effectiveness of mindfulness-based stress management treatment and the control group in decreasing anxiety.

Considering the calculated F-value in Table 6, i.e. $\mathrm{F}(27,1)=139.228, P<0.05)$, the obtained level of significance is 0.000 which is lower than 0.05 . Hence, the null hypothesis is rejected. Accordingly, it can be concluded that there is a significant difference between these two groups at the 95 confidence level and the third hypothesis is confirmed.

\section{Table 5. Descriptive data related to DASS-21.}

\begin{tabular}{cccccc}
\hline \multirow{2}{*}{ Indicators } & & \multicolumn{2}{c}{ Mindfulness group therapy } & \multicolumn{2}{c}{ Control group } \\
\cline { 3 - 6 } & & Mean & SD & Mean & SD \\
\hline \multirow{2}{*}{ Anxiety } & Pretest & $30 / 67$ & $2 / 69$ & $32 / 80$ & $2 / 48$ \\
& Posttest & $17 / 87$ & $2 / 87$ & $31 / 47$ & $7 / 91$ \\
\multirow{2}{*}{ Depression } & Pretest & $26 / 13$ & $3 / 15$ & $25 / 33$ & $4 / 11$ \\
& Posttest & $14 / 80$ & $3 / 36$ & $24 / 80$ & $3 / 44$ \\
\multirow{2}{*}{ Stress } & Pretest & $26 / 27$ & $4 / 52$ & $27 / 20$ & $3 / 69$ \\
& Posttest & $16 / 27$ & $2 / 81$ & $26 / 40$ & $2 / 74$ \\
\hline
\end{tabular}

Table 6. A summary of the results of covariance analysis of the results obtained from DASS-21.

\begin{tabular}{ccccccc}
\hline Resources of change & Sum of squares (type III) & df & Mean square & F & The probability level & Effect size \\
\hline Anxiety & 1067.007 & 1 & 1067.007 & 139.228 & 0.000 & 0.83 \\
Main effect & 16.546 & 1 & 16.546 & 2.159 & 0.153 & 0.07 \\
Residual error & 206.921 & 27 & & & & $0 / 000$ \\
Depression & $782 / 117$ & 1 & $782 / 117$ & $75 / 49$ & $0 / 047$ & $0 / 13$ \\
Main effect & $45 / 096$ & 1 & $45 / 096$ & $4 / 353$ & & $0 / 73$ \\
Residual error & $279 / 704$ & 27 & & & 0 & 0 \\
Stress & $743 / 251$ & 1 & $743 / 251$ & $94 / 446$ & $0 / 479$ & $0 / 019$ \\
Main effect & $4 / 055$ & 1 & $4 / 055$ & $0 / 515$ & &
\end{tabular}


Fourth Hypothesis: There is a significant difference between the effectiveness of mindfulness-based stress management treatment and the control group in decreasing depression.

Regarding the calculated F-value in Table 6, i.e. F $(27,1)=75.49, p<0.05)$, the obtained level of significance is 0.000 which is lower than 0.05 . Hence, the null hypothesis is rejected. Accordingly, it can be concluded that there is a significant difference between these two groups at the 95 confidence level and the fourth hypothesis is confirmed.

\section{Discussion and Conclusions}

First Hypothesis: There is a significant difference between the effectiveness of mindfulness-based stress management treatment and the control group considering craving beliefs.

The results associated with the first hypothesis indicated the effectiveness of the mindfulness group therapy, showing that this therapy was effective in reducing addicts' addiction craving. Since the control and the experimental groups' levels of addiction craving were different in the posttest, therefore, this hypothesis was confirmed. Accordingly, there was a significant difference between these two groups and the first hypothesis was confirmed.

Reviewing the existing literature demonstrated that this result was in line with the results of Hamedi et al. [9], Sterg et al. [11], Kiani et al. [13], Dabaghi et al. [18], Dabaghi et al. [28], Dabaghi [29], Bawen et al. [21], Garland [19], and Garland et al. [20].

Second Hypothesis: There is a significant difference between the effectiveness of mindfulness-based stress management treatment and the control group in reducing stress.

Third Hypothesis: There is a significant difference between the effectiveness of mindfulness-based stress management treatment and the control group in decreasing anxiety.

Fourth Hypothesis: There is a significant difference between the effectiveness of mindfulness-based stress management treatment and the control group in decreasing depression.

The results associated with the second hypothesis indicated the effectiveness of the mindfulness group therapy, showing that this therapy was effective in reducing addicts' stress. Since the control and the experimental groups' levels of stress were different in the posttest, therefore, this hypothesis was confirmed.

Moreover, through confirming the third and fourth hypotheses, it could be concluded that mindfulness group therapy was effective in reducing under treatment addicts' anxiety and depression.

Reviewing the existing literature demonstrated that this result was consistent with the results of Aghayousefi et al. [10] and Zarei et al. [12].

The results of previously conducted studies indicated that craving disrupted drug-dependents' attention [30]. Attentional bias is considered as a psychological component of craving which can be responsible for craving [31]. Considering the correlation between emotions (craving, depression, and anxiety) and cognition (attentional bias), it can be said that emotional states can adjust the focus and that attention processes can adjust emotional processes. Moreover, attentional bias adjusts symptoms related to drug abuse, relapse and craving [32].

As mentioned earlier, craving is significantly correlated with withdrawal symptoms and depressed/negative mood. A set of cognitive variables, including core beliefs, beliefs related to drug abuse, craving beliefs, and emotional regulation strategies, play a mediating role between negative emotions and relapse. Stress and anxiety are among emotional factors which can lead to an increase in drug abuse and its recurrence. Life stress is not only a risk factor in the addiction growth, but also a key factor in its recurrence.

According to what has been mentioned, it seems that the application of cognitive, meta-cognitive, and mindfulness techniques to deal with addiction craving and regulating emotions should be considered as an important part of a substance abuse treatment program. Mindfulness technique is an effective treatment which can alter the emotion regulation strategies. Therefore, considering these results, substance abuse treatment programs should aim at the mediating variables of consuming drugs.

Basically, in studies conducted on social problems, the applicability of the study should be considered. These studies should examine the variables which can be used in making appropriate educational programs aiming at changing and modifying behaviors and in operational decision-making targeted to fight with these substances to eliminate high risk factors and make appropriate decisions about treatment goals.

\section{References}

[1] Franken, I.H., Kroon, L.Y., Wiers, R.W. and Jansen, A. (2000) Selective Cognitive Processing of Drug Cues in Heroin 
Dependence. Journal of Psychopharmacology, 14, 395-400. http://dx.doi.org/10.1177/026988110001400408

[2] Tajeri, B. (2011) Addiction: Etiology and Treatment. Publication of New Ideas, Tehran.

[3] Alimadadi, A. (2005). A Drug Abuse Addiction Lesson According to the Chapters Presented by the Cultural Revolution Council. Jame' Negar Publication,Tehran.

[4] Daley, D.C. and Marlatt, G.A. (1992) Relapse Prevention: Cognitive and Behavioral Interventions. In: Lowinson, J.H., Ruiz, P., Millman, R.B. and Langrod, J.G., Eds., Substance Abuse: A Comprehensive Textbook, 2nd Edition, Williams \& Wilkins, Baltimore, 76-81.

[5] Kabat-Zinn, J. (1982) An Out-Patient Program in Behavioral Medicine for Chronic Pain Patients Based on the Practice of Mindfulness Meditation: Theoretical Considerations and Preliminary Results. General Hospital Psychiatry, 4, 3347. http://dx.doi.org/10.1016/0163-8343(82)90026-3

[6] Bowen, S., Chawla, N. and Marlatt, G.A. (2010) Mindfulness-Based Relapse Prevention for the Treatment of Substance Use Disorders: A Clinician's Guide. Guilford Press, New York.

[7] Witkiewitz, K., Marlatt, A. and Walker, D. (2005) Mindfulness-Based Relapse Prevention for Alcohol and Substance Use Disorders: The Meditation Tortoise Wins the Race. Journal of Cognitive Psychotherapy, 19, 229-221 http://dx.doi.org/10.1891/jcop.2005.19.3.211

[8] Leigh, J., Bowen, S. and Marlatt, G.A. (2005) Spirituality, Mindfulness and Substance Abuse. Addictive Behaviors, 30, 1335-1341. http://dx.doi.org/10.1016/j.addbeh.2005.01.010

[9] Hamedi, A., Shahidi, Sh. and Khademi, A. (2013) The Effectiveness of Mindfulness and Counseling on the Prevention of Recurrence of Drug Abuse. Addiction Research, 7, 101-118.

[10] Aghayousef, A.R., Oraki, M., Zare', M. and Imani, S. (2013) Mindfulness and Addiction: The Effectiveness of Mindfulness in Decreasing Stress, Anxiety, and Depression in Addicts. Journal of Behavior and Thought, 7, 27-36.

[11] Sterg, S., Kazemi, H. and Raeesi, Z. (2013) The Effectiveness of Cognitive Therapy on Craving Beliefs and Ideas Related to Patients Dependent to Industrial Drugs. Addiction Research, 7, 147-162.

[12] Zarei, S., Asadi, Z. and Zarei, Kh. (2014) The Effectiveness of Stress Management Training Program on Quality of Life Addicts Treated with Methadone. Journal of Addiction Research, 8, 9-19.

[13] Kiani, A.R., Ghasemi, N.O. and Pourabbas, A. (2012) Comparing the Effectiveness of Group Psychotherapy Based on Acceptance, Commitment, and Mindfulness in the Level of Addiction Craving and Cognitive Emotion Regulation in Methamphetamine Users. Journal of Addiction Research, 24, 27-38.

[14] Lak, Z., Moazedian, A., Hosseini Almadani, A., Sesaghat, M. and Ameri, S. (2012) The Effectiveness of Cognitive Behavioral Stress Management Training on Reducing Anxiety of Addicts Diagnosed with Generalized Anxiety Disorder. Journal of Addiction Research, 6, 69-84.

[15] Narimani, M. and Rajabi, S. (2012) The Effect of EEG Biofeedback on the Reduction of Depression, Anxiety, Stress and Craving Beliefs in Individuals with Substance Abuse Disorder. Journal of Addiction Research, 6, 7-18.

[16] Kiamarsi, A. and Abolghasemi, A. (2012) The Relationship of Self-Efficacy, Sensation Seeking and Coping Strategies with Aptitude of Substance Use in University Students. Journal of Addiction Research, 5, 7-24.

[17] Firozabadi, A., Gahnbari Hashemabadi, B.A. and Tabatabaee, M. (2009) The Effectiveness of Detached Mindfulness Techniques and Attention Distraction in the Level of Drug Cravings and the Possibility PF Relapse in Male Addicts. Principles of Mental Health, 11, 292-301.

[18] Dabaghi, P., Asgharnejad Farid, A.A., Atef Vahid, M.K. and Bolahri, J. (2007) The Effectiveness of Cognitive Therapy Based on Mindfulness and the Spiritual Schema Activation in Preventing Relapse in Opioid Abuse. Journal of Psychiatry and Clinical Psychology (Thinking and Behavior), 4, 366-375.

[19] Garland, E.L. (2011) Trait Mindfulness Predicts Attentional and Autonomic Regulation of Alcohol Cue-Reactivity. Journal of Psychophysiology, 25, 180-189. http://dx.doi.org/10.1027/0269-8803/a000060

[20] Garland, E.L., Boettiger, C.A., Gaylord, S., West, Chanon, V. and Howard, M.O. (2012) Mindfulness Is Inversely Associated with Alcohol Attentional Bias among Recovering Alcohol-Dependent Adults. Cognitive Therapy and Research, 36, 441-450. http://dx.doi.org/10.1007/s10608-011-9378-7

[21] Bowen, S.W., Chawla, N., Collins, S.E., Witkiewitz, K., Hsu, S., Grow, J. and Marlatt, G.A. (2009) Mindfulness-Based Relapse Prevention for Substance Use Disorders: A Pilot Efficacy Trial. Substance Abuse, 30, $295-305$. http://dx.doi.org/10.1080/08897070903250084

[22] Beck, A.T., Wright, F.D., Newman, C.F. and Liese, B.S. (1993) Cognitive Therapy of Substance Abuse. The Guilford Press. New York.

[23] Rahmanian, M., Mirja'fari, A. and Hassani, J. (2005) Examining the Attentional Bias towards Stimuli Related to Drugs in Patients Who Are Dependent to Opioid, Withdrew Opioid or Have Relapsed and Normal People. Journal of Psy- 
chology, 4, 413-428.

[24] Antony, M.M., Bieling, P.J., Cox, B.J., Enns, M.W. and Swinson, R.P. (1998) Psychometric Properties of the 42-Item and 21-Item Versions of the Depression Anxiety Stress Scales (DASS) in Clinical Groups and a Community Sample. Psychological Assessment, 10, 176-181. http://dx.doi.org/10.1037/1040-3590.10.2.176

[25] Lovibond, S.H. and Lovibond, P.F. (1995) Manual for the Depression Anxiety Stress Scales. 2nd Edition, Psychology Foundation, Sydney.

[26] Brown, T.A., Korotitsch, W., Chorpita, B.F. and Barlow, D.H. (1997) Psychometric Properties of the Depression Anxiety Stress Scales (DASS) in Clinical Samples. Behaviour Research and Therapy, 35, 79-89. http://dx.doi.org/10.1016/S0005-7967(96)00068-X

[27] Nunnally, J.C. and Bernstein, I.H. (1994) Psychometric Theory. McGraw-Hill, New York.

[28] Dabaghi, P., Asgharnejad Farid, A.A., Atef Vahid, M.K. and Bolahri, J. (2008) The Effectiveness of MindfulnessBased Relapse Prevention. Journal of Addiction Research, 2, 29-44.

[29] Dabaghi, P. (2007) The Effectiveness of Cognitive Therapy Based on Mindfulness and Artificial Activation Schemes in Preventing the Relapse in Opioid Abuse. PhD Thesis in Clinical Psychology, University of Medical Sciences, Tehran Psychiatric Institute, Tehran.

[30] Maude-Griffin, P.M. and Tiffany, S.T. (1996) Production of Smoking Urges through Imagery: The Impact of Affect and Smoking Abstinence. Experimental and Clinical Psychopharmacology, 4, 198-208. http://dx.doi.org/10.1037/1064-1297.4.2.198

[31] Waters, A.J. and Feyerabend, C. (2000) Determinants and Effects of Attentional Bias in Smokers. Psychology of Addictive Behaviors, 15, 268-271. http://dx.doi.org/10.1037/0893-164x.14.2.111

[32] Franken, I.H., Kroon, L.Y., Wiers, R.W. and Jansen, A. (2000) Selective Cognitive Processing of Drug Cues in Heroin Dependence. Journal of Psychopharmacology, 14, 395-400. http://dx.doi.org/10.1177/026988110001400408 